\title{
Serum markers for the early diagnosis of intestinal anastomotic leak after gyne-oncological operations
}

oguzhan kuru ${ }^{1}$, ilker cakir ${ }^{1}$, utku akgör ${ }^{1}$, serhat sen ${ }^{2}$, goksen gorgulu ${ }^{1}$, hasan emre ozdemir $^{1}$, Banu Isbilen Basok ${ }^{1}$, goksever akpinar ${ }^{1}$, and mehmet gokcu ${ }^{1}$

${ }^{1}$ Affiliation not available

${ }^{2}$ Izmir Katip Celebi Universitesi

May 1, 2021

\begin{abstract}
Objective: To analyze the serum markers for the early diagnosis of intestinal anastomotic leak (AL) after the gyne-oncological operations. Methods: Between September 2017 and March 2021, patients with an intestinal anastomosis performed during the gyne-oncological surgeries were identified from a tertiary center in Turkey. As the local guideline of the clinic, all these patients were followed by measuring serum samples including procalcitonin (PCT) and C-reactive protein (CRP) on postoperative day (POD) 1 through the day of discharge or the day of re-operation for AL. Results: $12.5 \%$ (5/40) of the patients suffered an AL and 4 of them were re-operated. The mean albumin values on POD 3-4 and the mean platelet values on POD 1 were lower in the AL group $(\mathrm{p}<0.05)$. Although it was not statistically significant $(\mathrm{p}>0.05)$, median PCT values $(\mathrm{ng} / \mathrm{mL})$ on POD 8-10 were higher in the AL group compared with no leak group. The best cutt-off point for PCT on POD 9 was determined to be $0.11 \mathrm{ng} / \mathrm{mL}$ (AUC: 0.917 , Sensitivity $=\% 100.0$, specifity $=\% 66.7$, positive predictive value $=\% 66.7$, negative predictive value $=\% 100.0)$. Conclusion: Serum PCT and CRP concentrations were not found to be helpfull for the early diagnosis of AL in patients operated for gyne-oncological malignancies. Low levels of albumin and platelets in the first days after the operation may be clue for a possible AL.
\end{abstract}

\section{Abstract}

Objective: To analyze the serum markers for the early diagnosis of intestinal anastomotic leak (AL) after the gyne-oncological operations.

Methods : Between September 2017 and March 2021, patients with an intestinal anastomosis performed during the gyne-oncological surgeries were identified from a tertiary center in Turkey. As the local guideline of the clinic, all these patients were followed by measuring serum samples including procalcitonin (PCT) and C-reactive protein (CRP) on postoperative day (POD) 1 through the day of discharge or the day of re-operation for AL.

Results : $12.5 \%(5 / 40)$ of the patients suffered an $\mathrm{AL}$ and 4 of them were re-operated. The mean albumin values on POD 3-4 and the mean platelet values on POD 1 were lower in the AL group $(\mathrm{p}<0.05)$. Although it was not statistically significant $(\mathrm{p}>0.05)$, median PCT values $(\mathrm{ng} / \mathrm{mL})$ on POD $8-10$ were higher in the AL group compared with no leak group. The best cutt-off point for PCT on POD 9 was determined to be $0.11 \mathrm{ng} / \mathrm{mL}$ (AUC: 0.917 , Sensitivity $=\% 100.0$, specifity $=\% 66.7$, positive predictive value $=\% 66.7$, negative predictive value $=\% 100.0)$.

Conclusion: Serum PCT and CRP concentrations were not found to be helpfull for the early diagnosis of $\mathrm{AL}$ in patients operated for gyne-oncological malignancies. Low levels of albumin and platelets in the first days after the operation may be clue for a possible AL. 
Keywords : Procalcitonin; Anastomotic leak; Early diagnosis.

\section{What's already known about this topic?}

Previous studies have shown that inflammatory biomarkers like procalcitonin and C-reactive protein may be useful in the early diagnosis of anastomotic leakage after major surgeries.

\section{What does this article add?}

Serum procalcitonin and C-reactive protein concentrations were not found to be helpful for the early diagnosis of AL in patients operated for gyne-oncological malignancies. Low levels of albumin and platelets in the first days after the operation may be a clue for a possible anastomotic leakage.

\section{INTRODUCTION}

Anastomotic leak (AL) after intestinal surgery has a dramatical impact on patient's outcome regarding its high morbidity $(35 \%)$ and variable mortality $(4-22 \%)^{1,2}$.Although early diagnosis and immediate intervention improves the prognosis, in many patients, due to non-specific symptoms and indeterminate radiologic imaging, clinical diagnosis of AL may delay untill 6-12 days after surgery ${ }^{3}$.

Previous studies have shown that inflammatory biomarkers like procalcitonin (PCT) and C-reactive protein (CRP) may be useful in the early diagnosis of AL after major surgeries ${ }^{4}$. But, up to now, no studies evaluating the role of these markers in the early detection of AL after gyne-oncological operations have been found in the literature.

In the present study, we aimed to analyze the serum markers for the early diagnosis of AL after the gyneoncological operations.

\section{MATERIALS AND METHODS}

\section{Study design and patient selection}

After obtaining the approval of institutional review board (Approval no: 2020/8-2), a retrospective review of the patients who underwent an intestinal anastomosis during the gyne-oncological surgeries between September 2017 and March 2021 was performed at an ESGO (European Society of Gynecological Oncology) accredited center in Turkey (Tepecik Education and Research Hospital, Izmir). Patients' hospital files, pathology and radiology reports, and laboratory results were throughly reviewed. Exclusion criteria were defined as R2 cancer resection (gross residual disease) and non-gynecological malignancies according to final pathology report. Collected data included patient's age, body mass index (BMI) $\left(\mathrm{kg} / \mathrm{m}^{2}\right)$, ASA (American Society of Anesthesiologists) score, type of the malignancy, operative procedure, patient outcome, and the serum levels of biomarkers taken through the hospitalization time.

\section{Clinical management}

Antibiotic prophylaxis with cefazolin was given 30 minutes before the incision and repeated if the operation lasted more than 3 hours. Combined antibiotic therapy (cefazolin plus metronidazole) continued untill the day of discharge. On the day before and the morning of the operation, Fleet Enema $133 \mathrm{~mL}$ rectally was applied. Abdominal catheter was placed in all of the patients and the drain was taken out after the patient tolerated oral intake. Nasogastric catheter was holded in the first 24 hours. Oral intake was restricted in the first 72 hours and started as liquid intake and progressed day by day with the status of the bowel movements and the gas discharge. AL was suspected if the patient had fever and/or abdominal tenderness and/or fecal drainage from the drain/incision/vaginal cuff. All ALs were determined either by a contrast-enhanced computed tomography (CT) and/or intraoperative findings. Clavien-Dingo classification was used to define the AL as major (need of re-operation) or minor (conservative management) ${ }^{5}$. In statistical analysis, major and minor ALs were analyzed in the same group. Patients were allowed to discharge if they had oral intake without vomiting, feces discharge, and no sign of peritonitis.

\section{Analysis of PCT and CRP}


In the study period, according to local clinical guideline, postoperative daily serum samples including PCT, CRP, leukocytes, neutrophils, platelets, albumin were collected from postoperative day (POD) 1 to the day of discharge or the day of re-operation for AL.

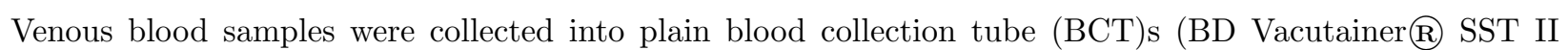
Advance Tube, $5 \mathrm{~mL}, 13 \times 100 \mathrm{~mm}$, USA). Serum samples were separated from cellular fragments by centrifugation for 10 minutes at $1.500 \mathrm{~g}$ within one hour after blood sampling. Serum PCT levels were measured by the sandwich immunoassay using an immunoassay analyzer (Advia Centaur XP, Siemens Healthineers, Erlangen, Germany). The functional assay sensitivity of the Advia Centaur BRAHMS PCT is $0.05 \mathrm{ng} / \mathrm{mL}$ with an analytical range as $0.02-75 \mathrm{ng} / \mathrm{mL}$. The reference range for a healthy adult population is lower than $0.1 \mathrm{ng} / \mathrm{mL}$. A value of $2 \mathrm{ng} / \mathrm{mL}$ represents a high risk of severe sepsis or septic shock.

CRP levels were measured by a latex-immunoturbidimetric method with an AU5800 (Beckman Coulter Inc., USA) analyzer. The analytical range of the test is between 0.2 to $480 \mathrm{mg} / \mathrm{L}$. The reference range for a healthy adult population is lower than $5 \mathrm{mg} / \mathrm{L}$.

\section{Statistical analysis}

The study data were subjected to statistical analysis using the SPSS version 20.0 (SPSS Inc, Chicago, IL). All data were expressed as numbers, percentiles, mean, median, and standard deviation (SD). Student t test was used for univariate analysis of the difference between groups. Mann-Whitney U test was performed as the non-parametric test. The receiving operating characteristic (ROC) curve and the value of area under the curve (AUC) were used to analyze the accuracy of different parameters to predict AL and the best cut-off point was selected. $\mathrm{p}<0.05$ was considered statistically significant.

\section{RESULTS}

The study population consisted of 40 patients. Mean age was $52.3+-12.3$ years. The most common type of malignancy was ovarian cancer $(70.0 \%) .78 .5 \%$ of the patients were operated for primary debulking purpose. $12.5 \%(5 / 40)$ of the patients suffered an AL. The clinical characteristics of the study population were shown in Table 1.

The most common operation was rectosigmoid resection $(67.5 \%)$. The mean operation time was $367.6+-$ 122.3 minutes. A protective ostomy was opened in $7.5 \%$ (3/40) of the patients. Anastomosis was most frequently performed with stapler devices $(33 / 40,82.5 \%)$. In addition to intestinal anastomosis; peritonectomy, splenectomy, appendectomy, and lymph node (pelvic and/or paraaortic) resection were also performed in $57.5 \%, 10.0 \%, 50.0 \%, 67.5 \%$ of the patients, respectively (Table 2 ).

In both groups, PCT values tended to decrease until POD 7. Although the PCT values started to increase from the POD 7 through POD 8-9 in the leak group, they remained stable in the no leak group. The albumin decrease starting from POD 1 to POD 4 was observed in the leak group while it remained nearly stable in the no leak group through the first 10 days postoperatively. The change of mean PCT and albumin values in anastomotic leak and no leak groups within 10 days postoperatively was shown in Figures 1 and 2 .

Postoperative 10-day PCT, albumin and platelet values were compared in the group with and without AL. There was a statistically significant difference between the albumin values on POD $3(\mathrm{p}=0.028)$, on POD $4(\mathrm{p}=0.045)$ and the platelet values on POD $1(\mathrm{p}<0.001)$. The mean albumin values on POD $3-4$ and the mean platelet values on POD 1 were lower in the AL group. Although it was not statistically significant $(\mathrm{p}>0.05)$, median PCT values $(\mathrm{ng} / \mathrm{mL})$ on POD 8,9 and 10 were higher in the AL group compared with no leak group (Table 3). The best cutt-off point for PCT on POD 9 was determined to be $0.11 \mathrm{ng} / \mathrm{mL}$ (AUC: 0.917 , Sensitivity $=\% 100.0$, specifity $=\% 66.7$, positive predictive value $=\% 66.7$, negative predictive value $=\% 100.0)$. Postoperative 10-day CRP, leukocyte, neutrophil, leukocyte ratio neutrophil (L/N) and fever values were also compared in the group with and without AL, and no statistically significant difference was found $(\mathrm{p}>0.05)$ (not shown in the table).

In 4 of 5 patients with AL, peritonectomy was added to the debulking procedure. Due to severe peritonitis, 
4 patients were re-operated on POD 8-10 and the leakage was classified as major. The other patient was discharged on POD 10 before the diagnosis of AL, and re-hospitalized on POD 15 for a rectovaginal fistula. That patient was followed conservatively up to 2 months untill the fistula closed on its own which provided to classify the leakage as minor. One of the patients with AL died of multiorgan failure on POD 20. That patient was operated for endometrial carcinosarcoma and in the removal of omental cake, transverse colectomy and anastomosis was performed with handsewn technique. Clinical and surgical details of the 5 patients with AL were detailed in Table 4.

\section{DISCUSSION}

Our study is the first study evaluating the role of serum markers in the early detection of AL after gyneoncological operations. There have been many studies showing the possible role of serum PCT and CRP in the diagnosis of AL after elective colorectal surgeries ${ }^{6}$. In the study of Alvara Garcia-Granero et al., in case of major AL, PCT and CRP were proved as reliable predictors on POD 3 to 5 with the best value for $\mathrm{PCT}$ on POD 5 was $0.31 \mathrm{ng} / \mathrm{ml}(\mathrm{AUC}=0.86)$ (Sensitivity $=100 \%$, specifity $=72 \%$, positive predictive value $=17 \%$, negative predictive value $=100 \%)^{7}$. Also, in a recent meta-analysis, it was concluded that $\mathrm{PCT}$ is a useful negative test for AL after elective colorectal surgery with negative predictive values ranging from $95 \%$ to $100 \%$ (highest AUROC was 0.88 on POD 5). But, as an isolated test, poor positive predictive values of up to $34 \%$, limit its use in predicting $\mathrm{AL}^{8}$. Different from elective colorectal surgeries, in the surgical concept of gynecological malignancies (especially in advanced ovarian cancer), debulking procedures including both upper and lower abdomen and peritonectomy were needed to reach zero macroscopic residual disease to get overall survival advantage ${ }^{9,10}$. So, we wanted to analyze these markers whether they were also beneficial in the early diagnosis of AL after gyne-oncological surgeries. And, a statistically significant difference was found between the albumin values on POD $3(\mathrm{p}=0.028)$, on POD $4(\mathrm{p}=0.045)$ and the platelet values on POD $1(\mathrm{p}<0.001)$. Pre-operative hypoalbuminemia is a well-known risk factor for AL, but few studies evaluated the role of peri-operative albumin on $\mathrm{AL}$ after intestinal surgeries ${ }^{11,12}$. In the study of Shimura et al., it was shown that lower average levels of serum albumin on POD 1 and POD 3 (HR $=4.49$, $95 \% \mathrm{CI}=0.77-29.58 ; \mathrm{p}=0.0955)$ and higher average levels of serum leukocytes on POD 1 and POD 3 $(\mathrm{HR}=5.62,95 \% \mathrm{CI}=0.76-115.34 ; p=0.0952)$ were independent risk factors for $\mathrm{AL}^{11}$. Similarly, in our study, lower levels of albumin on POD 3 and 4 were associated with AL. In the study of Margarson et al., after giving intravenous hypertonic albumin solution, serum albumin concentrations decrease significantly faster in septic patients than in healthy controls and that was explained by increased vascular leak of the albumin $^{13}$. But, it is unclear whether hypoalbuminemia is the cause or result of the AL.

Interestingly, in our study, it was shown for the first time in the literature that platelet count was associated with AL as the mean platelet values were lower on POD 1 in the AL group. In the study of Dewitte et al, possible mechanisms of blood platelets in sepsis pathophysiology were reviewed ${ }^{14}$. According to this, beyond their roles in haemostasis, platelets are now accepted as active actors of immune respone playing role in host defence and tissue integrity. This interesting relation of low platelets with AL found in our study should be further evaluated in prospective trials.

Although it was not statistically significant ( $\mathrm{p}>0.05$ ), in our study, median PCT values on POD 8 to 10 were higher in the AL group. And, PCT values increased later compared to other studies in the literature ${ }^{7,15}$. Intraabdominal sepsis may have delayed in patients with AL due to our local peroperative guideline which was different from ERAS protocol ${ }^{16}$. Most likely reason for this late increase of PCT in patients with AL may be antibiotic supression during our hospitalization period. In accord to this, Charles et al. showed that empirical antibiotic therapy was associated with a greater decline in PCT following the onset of sepsis between day 2 and $3^{17}$.

In contrast to previous studies, we could not find a statistically significance between AL and postoperative CRP levels ${ }^{7,18}$. In a systematic review made by Singh et al., it was shown that serum CRP level on POD 3,4 , and 5 had comparable diagnostic accuracy for the prediction of AL with a AUROC of $0.81,0.80$, and 0.80 , respectively ${ }^{18}$. The derived CRP cut-off values were $172 \mathrm{mg} / \mathrm{L}$ on POD $3,124 \mathrm{mg} / \mathrm{L}$ on POD 4 and $144 \mathrm{mg} / \mathrm{L}$ on POD 5. And these corresponded to a negative predictive value of $97 \%$ and a positive 
predictive value of $21-23 \%{ }^{18}$. In the study of Smith et al., for the first time in the literature, an association of biomarker trajectory was assessed, instead of isolated daily values. And the trend of CRP for the first 5 days following surgery, appeared to be highly accurate for diagnosing AL, with a daily rise of 50 units had a sensitivity of $91 \%$ and a negative predictive value of $99.3 \%{ }^{19}$. Our results showed that PCT and CRP did not work to predict AL before clinical onset of sepsis in patients operated for gyne-oncological surgeries. After ROC analysis, the best cutt-off point for PCT was determined to be $0.11 \mathrm{ng} / \mathrm{mL}$ on POD 9. Although this statistical value was not useful for the early clinical management, it could be used in patients who had vague symptoms and indeterminate radiologic imaging. Our low number of positive cases (5 cases of AL) may have prevented us from observing possible effect of PCT and CRP on the early days of leakage.

Our study had some limitations. First, the study had retrospective nature comprising consecutive patients managed on the basis of local clinical guideline. Second, patient cohort included heteregoneus type of malignancies and surgeries. On the other hand, PCT, previously shown to predict AL in colorectal surgery, has been shown for the first time to be useless in gynecological oncology. Also, different from literature, the increase of PCT seen in AL appeared very soon that should be supported with further studies.

In conclusion, serum PCT and CRP concentrations were not found to be helpfull for the early diagnosis of $\mathrm{AL}$ in patients operated for gyne-oncological malignancies. Low levels of albumin and platelets in the first days after the operation may be clue for a possible AL.

\section{Conflict of Interest}

The authors have no conflicts of interest.

\section{Author contribution}

Data curation: I.C., G.G., H.E.O.

Supervision: M.G., B.I.B.

Writing - review \& editing: O.K., U.A.

Investigation: G.A., S.S.

\section{REFERENCES}

1. Alberts JC, Parvaiz A, Moran BJ. Predicting risk and diminishing the consequences of anastomotic dehiscence following rectal resection. Colorectal disease : the official journal of the Association of Coloproctology of Great Britain and Ireland 2003; 5 (5): 478-82.

2. Alves A, Panis Y, Mathieu P, Mantion G, Kwiatkowski F, Slim K. Postoperative mortality and morbidity in French patients undergoing colorectal surgery: results of a prospective multicenter study.Archives of surgery (Chicago, Ill : 1960) 2005; 140 (3): 278-83, discussion 84.

3. Kurman RJ, Carcangiu ML, Herrington CS. World Health Organisation Classification of Tumours of the Female Reproductive Organs: International Agency for Research on Cancer; 2014.

4. Aiolfi A, Asti E, Rausa E, Bonavina G, Bonitta G, Bonavina LJPO. Use of C-reactive protein for the early prediction of anastomotic leak after esophagectomy: Systematic review and Bayesian meta-analysis. 2018;13 (12): e0209272.

5. Clavien PA, Barkun J, de Oliveira ML, et al. The Clavien-Dindo classification of surgical complications: five-year experience. Annals of surgery 2009; 250 (2): 187-96.

6. Chernyshov S, Alexeev M, Rybakov E, et al. Risk factors and inflammatory predictors for Anastomotic Leakage following Total Mesorectal Excision with defunctioning stoma. Pol Przegl Chir2018; 90 (3): 31-6.

7. Garcia-Granero A, Frasson M, Flor-Lorente B, et al. Procalcitonin and C-reactive protein as early predictors of anastomotic leak in colorectal surgery: a prospective observational study. Diseases of the colon and rectum 2013; 56 (4): 475-83. 
8. Su'a B, Tutone S, MacFater W, et al. Diagnostic accuracy of procalcitonin for the early diagnosis of anastomotic leakage after colorectal surgery: a meta-analysis. ANZ journal of surgery 2020;90 (5): 675-80.

9. Colombo N, Sessa C, du Bois A, et al. ESMO-ESGO consensus conference recommendations on ovarian cancer: pathology and molecular biology, early and advanced stages, borderline tumours and recurrent disease+.Annals of oncology : official journal of the European Society for Medical Oncology 2019; 30 (5): 672-705.

10. Rodriguez N, Miller A, Richard SD, et al. Upper abdominal procedures in advanced stage ovarian or primary peritoneal carcinoma patients with minimal or no gross residual disease: an analysis of Gynecologic Oncology Group (GOG) 182. Gynecologic oncology 2013;130 (3): 487-92.

11. Shimura T, Toiyama Y, Hiro J, et al. Monitoring perioperative serum albumin can identify anastomotic leakage in colorectal cancer patients with curative intent. Asian journal of surgery 2018;41 (1): 30-8.

12. Wang XT, Kong FB, Mai W, Li L, He CG. Monitoring perioperative serum albumin can identify anastomotic leakage in colorectal cancer patients with curative intent. Asian journal of surgery 2019;42 (2): $472-3$.

13. Margarson MP, Soni NC. Changes in serum albumin concentration and volume expanding effects following a bolus of albumin $20 \%$ in septic patients. British journal of anaesthesia 2004; 92 (6): 821-6.

14. Dewitte A, Lepreux S, Villeneuve J, et al. Blood platelets and sepsis pathophysiology: A new therapeutic prospect in critically [corrected] ill patients? Ann Intensive Care 2017;7 (1): 115-.

15. Lagoutte N, Facy O, Ravoire A, et al. C-reactive protein and procalcitonin for the early detection of anastomotic leakage after elective colorectal surgery: pilot study in 100 patients. Journal of visceral surgery 2012; 149 (5): e345-9.

16. Nygren J, Thacker J, Carli F, et al. Guidelines for perioperative care in elective rectal/pelvic surgery: Enhanced Recovery After Surgery (ERAS(r)) Society recommendations. Clinical nutrition (Edinburgh, Scotland) 2012; 31 (6): 801-16.

17. Charles PE, Tinel C, Barbar S, et al. Procalcitonin kinetics within the first days of sepsis: relationship with the appropriateness of antibiotic therapy and the outcome. Critical care (London, England) 2009; 13 (2): R38.

18. Singh PP, Zeng IS, Srinivasa S, Lemanu DP, Connolly AB, Hill AG. Systematic review and meta-analysis of use of serum C-reactive protein levels to predict anastomotic leak after colorectal surgery. The British journal of surgery 2014; 101 (4): 339-46.

19. Smith SR, Pockney P, Holmes R, et al. Biomarkers and anastomotic leakage in colorectal surgery: C-reactive protein trajectory is the gold standard. ANZ journal of surgery 2018; 88 (5): 440-4.

\section{Figure Legends}

Figure 1. Mean procalcitonin change in anastomotic leak and no leak groups within 10 days postoperatively. $\mathrm{POD}=$ Postoperative day

Figure 2. Mean albumin change in anastomotic leak and no leak groups within 10 days postoperatively.

$\mathrm{POD}=$ Postoperative day

\section{Table Legends}

Table 1 . Clinical characteristics of the study population.

$\mathrm{SD}=$ Standard deviation; $\mathrm{n}=$ Number; $\%=$ Column percent; $\mathrm{CT}=$ Chemotherapy; CRT $=$ Chemoradiotherapy. 
Table 2. Surgical data of the study population.

$\mathrm{SD}=$ Standard deviation; $\mathrm{n}=$ Number; $\%=$ Column percent

Table 3. Comparison of serum markers within 10 days postoperatively in the group with and without anastomotic leakage.

$\mathrm{POD}=$ Postoperative day; $\mathrm{SD}=$ Standard deviation; $\mathrm{p}=$ Mann-Whitney $\mathrm{U}$ test,${ }^{*}=$ Student $\mathrm{t}$ test

Table 4 . Clinical and surgical details of the 5 patients with anastomotic leakage.

$\mathrm{POD}=$ Postoperative day $\mathrm{AL}=$ Anastomotic leakage $\mathrm{App}=$ Appendectomy; Per = Peritonectomy; Ppa $=$ Pelvic-paraaortic lymph node resection; $\mathrm{RS}=$ Rectosigmoid resection;

$\mathrm{TC}=$ Transverse colectomy; TJ = Total subcolectomiy plus jejunoileal resection; $\mathrm{S}=$ Stapler, $\mathrm{H}=\mathrm{Hand}-$ sewn; GS = General surgeon; GO = Gyne-oncolog.

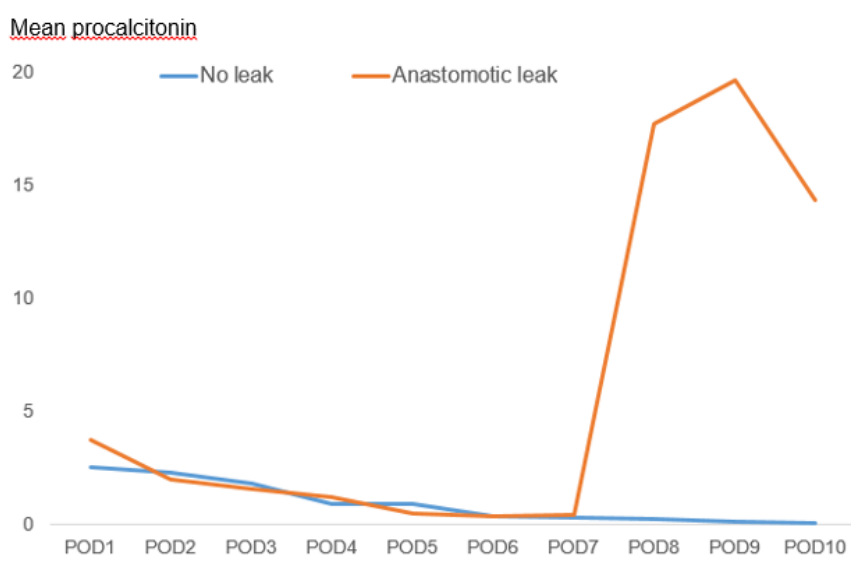




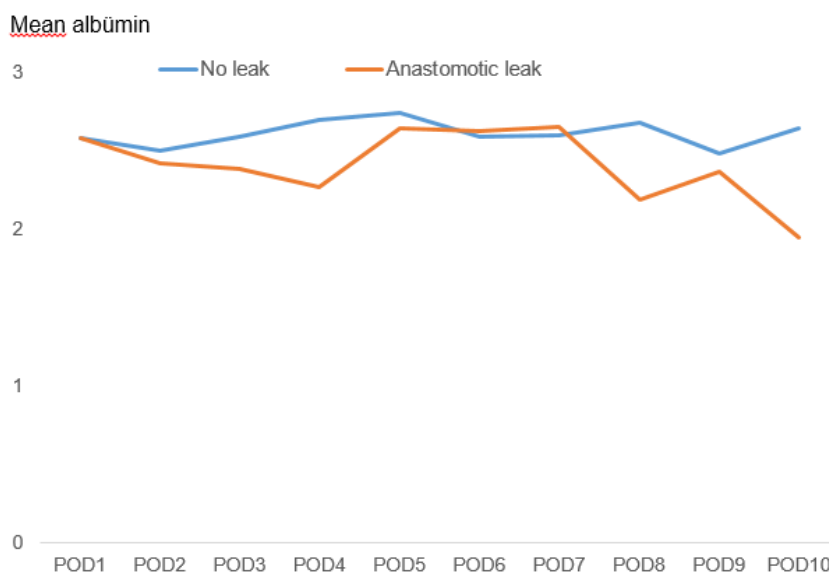

$\begin{array}{lllllllll}\text { POD1 POD2 } & \text { POD3 } & \text { POD4 } & \text { POD5 } & \text { POD6 } & \text { POD7 } & \text { POD8 } & \text { POD9 } & \text { POD10 }\end{array}$

\section{Hosted file}

Tables-New.pdf available at https://authorea.com/users/411297/articles/520384-serum-markersfor-the-early-diagnosis-of-intestinal-anastomotic-leak-after-gyne-oncological-operations 